\title{
Multiple Positive Solutions of Fourth-Order Impulsive Differential Equations with Integral Boundary Conditions and One-Dimensional $p$-Laplacian
}

\author{
Meiqiang Feng \\ School of Applied Science, Beijing Information Science E Technology University, Beijing 100192, China \\ Correspondence should be addressed to Meiqiang Feng, meiqiangfeng@sina.com \\ Received 2 February 2010; Revised 25 April 2010; Accepted 5 June 2010 \\ Academic Editor: Gennaro Infante \\ Copyright (C) 2011 Meiqiang Feng. This is an open access article distributed under the Creative \\ Commons Attribution License, which permits unrestricted use, distribution, and reproduction in \\ any medium, provided the original work is properly cited. \\ By using the fixed point theory for completely continuous operator, this paper investigates the \\ existence of positive solutions for a class of fourth-order impulsive boundary value problems \\ with integral boundary conditions and one-dimensional $p$-Laplacian. Moreover, we offer some \\ interesting discussion of the associated boundary value problems. Upper and lower bounds for \\ these positive solutions also are given, so our work is new.
}

\section{Introduction}

The theory of impulsive differential equations describes processes which experience a sudden change of their state at certain moments. Processes with such a character arise naturally and often, especially in phenomena studied in physics, chemical technology, population dynamics, biotechnology, and economics. For an introduction of the basic theory of impulsive differential equations, see Lakshmikantham et al. [1]; for an overview of existing results and of recent research areas of impulsive differential equations, see Benchohra et al. [2]. The theory of impulsive differential equations has become an important area of investigation in recent years and is much richer than the corresponding theory of differential equations (see, e.g., [3-18] and references cited therein).

Moreover, the theory of boundary-value problems with integral boundary conditions for ordinary differential equations arises in different areas of applied mathematics and physics. For example, heat conduction, chemical engineering, underground water flow, thermo-elasticity, and plasma physics can be reduced to the nonlocal problems with integral boundary conditions. For boundary-value problems with integral boundary conditions and 
comments on their importance, we refer the reader to the papers by Gallardo [19], Karakostas and Tsamatos [20], Lomtatidze and Malaguti [21], and the references therein. For more information about the general theory of integral equations and their relation with boundaryvalue problems, we refer to the book of Corduneanu [22] and Agarwal and O'Regan [23].

On the other hand, boundary-value problems with integral boundary conditions constitute a very interesting and important class of problems. They include two, three, multipoint and nonlocal boundary-value problems as special cases. The existence and multiplicity of positive solutions for such problems have received a great deal of attention in the literature. To identify a few, we refer the reader to [24-46] and references therein. In particular, we would like to mention some results of Zhang et al. [34], Kang et al. [44], and Webb et al. [45]. In [34], Zhang et al. studied the following fourth-order boundary value problem with integral boundary conditions

$$
\begin{gathered}
x^{(4)}(t)-\lambda f(t, x(t))=\theta, \quad 0<t<1, \\
x(0)=x(1)=\int_{0}^{1} g(s) x(s) d s, \\
x^{\prime \prime}(0)=x^{\prime \prime}(1)=\int_{0}^{1} h(s) x(s) d s,
\end{gathered}
$$

where $\mathcal{\lambda}$ is a positive parameter, $f \in C([0,1] \times P, P), \theta$ is the zero element of $E$, and $g, h \in$ $L^{1}[0,1]$. The authors investigated the multiplicity of positive solutions to problem (1.1) by using the fixed point index theory in cone for strict set contraction operator.

In [44], Kang et al. have improved and generalized the work of [34] by applying the fixed point theory in cone for a strict set contraction operator; they proved that there exist various results on the existence of positive solutions to a class of fourth-order singular boundary value problems with integral boundary conditions

$$
\begin{gathered}
u^{(4)}(t)=a(t) f\left(t, u(t),-u^{\prime \prime}(t)\right)+b(t) g\left(t, u(t),-u^{\prime \prime}(t)\right), \quad 0<t<1, \\
a_{1} u(0)-b_{1} u^{\prime}(0)=\int_{0}^{1} m_{1}(s) u(s) d s, \quad c_{1} u(1)+d_{1} u^{\prime}(1)=\int_{0}^{1} n_{1}(s) u(s) d s, \\
a_{2} u^{\prime \prime}(0)-b_{2} u^{\prime \prime \prime}(0)=\int_{0}^{1} m_{2}(s) u^{\prime \prime}(s) d s, \quad c_{2} u^{\prime \prime}(2)+d_{2} u^{\prime \prime \prime}(1)=\int_{0}^{1} n_{2}(s) u^{\prime \prime}(s) d s,
\end{gathered}
$$

where $a, b \in C((0,1),[0,+\infty))$ and may be singular at $t=0$ or $t=1 ; f, g:(0,1) \times P \backslash\{\theta\} \times$ $P \backslash\{\theta\} \rightarrow P$ are continuous and may be singular at $t=0,1, u=0$, and $u^{\prime \prime}=0 ; a_{i}, b_{i}, c_{i}$, and $d_{i} \in[0,+\infty)$, and $\rho_{i}=a_{i} c_{i}+a_{i} d_{i}+b_{i} c_{i}>0$, and $m_{i}, n_{i} \in L^{1}[0,1]$ are nonnegative, $i=1,2$.

More recently, by using a unified approach, Webb et al. [45] considered the widely studied boundary conditions corresponding to clamped and hinged ends and many nonlocal boundary conditions and established excellent existence results for multiple positive solutions of fourth-order nonlinear equations which model deflections of an elastic beam

$$
u^{4}(t)=g(t) f(t, u(t)), \quad \text { for almost every } t \in(0,1)
$$


subject to various boundary conditions

$$
\begin{gathered}
u(0)=0, \quad u(1)=\alpha[u], \quad u^{\prime}(0)=0, \quad u^{\prime}(1)=0, \\
u(0)=0, \quad u(1)=0, \quad u^{\prime}(0)=0, \quad u^{\prime}(1)+\alpha[u]=0, \\
u(0)=0, \quad u(1)=\alpha[u], \quad u^{\prime \prime}(0)=0, \quad u^{\prime \prime}(1)=0, \\
u(0)=0, \quad u(1)=0, \quad u^{\prime \prime}(0)=0, \quad u^{\prime \prime}(1)+\alpha[u]=0,
\end{gathered}
$$

where $\alpha[u]$ denotes a linear functional on $C[0,1]$ given by $\alpha[u]=\int_{0}^{1} u(s) d A(s)$ involving a Stieltjes integral, and $A$ is a function of bounded variation.

At the same time, we notice that there has been a considerable attention on $p$-Laplacian BVPs $[18,32,35,36,38,42]$ as $p$-Laplacian appears in the study of flow through porous media $(p=3 / 2)$, nonlinear elasticity $(p \geq 2)$, glaciology $(1 \leq p \leq 4 / 3)$, and so forth. Here, it is worth mentioning that Liu et al. [43] considered the following fourth-order four-point boundary value problem:

$$
\begin{aligned}
\left(\phi_{p}\left(x^{\prime \prime}(t)\right)\right)^{\prime \prime} & =w(t) f(t, x(t)), \quad t \in[0,1], \\
x(0) & =0, \quad x(1)=a x(\xi), \\
x^{\prime \prime}(0) & =0, \quad x^{\prime \prime}(1)=b x^{\prime \prime}(\eta),
\end{aligned}
$$

where $0<\xi, \eta<1,0 \leq a<b<1$, and $f \in C((0,1) \times(0,+\infty),[0,+\infty))$. By using upper and lower solution method, fixed-point theorems, and the properties of Green's function $G(t, s)$ and $H(t, s)$, the authors give sufficient conditions for the existence of one positive solution.

Motivated by works mentioned above, in this paper, we consider the existence of positive solutions for a class of boundary value problems with integral boundary conditions of fourth-order impulsive differential equations:

$$
\begin{gathered}
\left(\phi_{p}\left(y^{\prime \prime}(t)\right)\right)^{\prime \prime}=f(t, y(t)), \quad t \in J, t \neq t_{k}, k=1,2, \ldots, m, \\
\left.\Delta y^{\prime}\right|_{t=t_{k}}=-I_{k}\left(y\left(t_{k}\right)\right), \quad k=1,2, \ldots, m, \\
y(0)=y(1)=\int_{0}^{1} g(t) y(t) d t, \\
\phi_{p}\left(y^{\prime \prime}(0)\right)=\phi_{p}\left(y^{\prime \prime}(1)\right)=\int_{0}^{1} h(t) \phi_{p}\left(y^{\prime \prime}(t)\right) d t .
\end{gathered}
$$

Here $J=[0,1], \phi_{p}(s)$ is $p$-Laplace operator, that is, $\phi_{p}(s)=|s|^{p-2} s, p>1,\left(\phi_{p}\right)^{-1}=\phi_{q}, 1 / p+$ $1 / q=1, f \in C\left(J \times R^{+}, R^{+}\right), I_{k} \in C\left(R^{+}, R^{+}\right), R^{+}=[0,+\infty), t_{k}(k=1,2, \ldots, m)$ (where $m$ is fixed positive integer) are fixed points with $0=t_{0}<t_{1}<t_{2}<\cdots<t_{k}<\cdots<t_{m}<t_{m+1}=1$, $\left.\Delta x^{\prime}\right|_{t=t_{k}}=x^{\prime}\left(t_{k}^{+}\right)-x^{\prime}\left(t_{k}^{-}\right)$, where $x^{\prime}\left(t_{k}^{+}\right)$and $x^{\prime}\left(t_{k}^{-}\right)$represent the right-hand limit and left-hand limit of $x^{\prime}(t)$ at $t=t_{k}$, respectively, and $g, h \in L^{1}[0,1]$ is nonnegative.

For the case of $I_{k}=0, k=1,2, \ldots, m$, problem (1.6) reduces to the problem studied by Zhang et al. in [33]. By using the fixed point theorem in cone, the authors obtained some 
sufficient conditions for the existence and multiplicity of symmetric positive solutions for a class of $p$-Laplacian fourth-order differential equations with integral boundary conditions.

For the case of $I_{k}=0, k=1,2, \ldots, m, g=0, h=0$, and $p=2$, problem (1.6) is related to fourth-order two-points boundary value problem of ODE. Under this case, problem (1.6) has received considerable attention (see, e.g., [40-42] and references cited therein). Aftabizadeh [40] showed the existence of a solution to problem (1.6) under the restriction that $f$ is a bounded function. Bai and Wang [41] have applied the fixed point theorem and degree theory to establish existence, uniqueness, and multiplicity of positive solutions to problem (1.6). Ma and Wang [42] have proved that there exist at least two positive solutions by applying the existence of positive solutions under the fact that $f(t, u)$ is either superlinear or sublinear on $u$ by employing the fixed point theorem of cone extension or compression.

Being directly inspired by $[18,34,43]$, in the present paper, we consider some existence results for problem (1.6) in a specially constructed cone by using the fixed point theorem. The main features of this paper are as follows. Firstly, comparing with [39-43], we discuss the impulsive boundary value problem with integral boundary conditions, that is, problem (1.6) includes fourth-order two-, three-, multipoint, and nonlocal boundary value problems as special cases. Secondly, the conditions are weaker than those of $[33,34,46]$, and we consider the case $I_{k} \neq 0$. Finally, comparing with $[33,34,39-43,46]$, upper and lower bounds for these positive solutions also are given. Hence, we improve and generalize the results of $[33,34,39$ $43,46]$ to some degree, and so, it is interesting and important to study the existence of positive solutions of problem (1.6).

The organization of this paper is as follows. We shall introduce some lemmas in the rest of this section. In Section 2, we provide some necessary background. In particular, we state some properties of the Green's function associated with problem (1.6). In Section 3, the main results will be stated and proved. Finally, in Section 4, we offer some interesting discussion of the associated problem (1.6).

To obtain positive solutions of problem (1.6), the following fixed point theorem in cones is fundamental, which can be found in [47, page 94].

Lemma 1.1. Let $\Omega_{1}$ and $\Omega_{2}$ be two bounded open sets in Banach space $E$, such that $0 \in \Omega_{1}$ and $\bar{\Omega}_{1} \subset \Omega_{2}$. Let $P$ be a cone in $E$ and let operator $A: P \cap\left(\bar{\Omega}_{2} \backslash \Omega_{1}\right) \rightarrow P$ be completely continuous. Suppose that one of the following two conditions is satisfied:

(a) $\|A x\| \geq\|x\|$, for all $x \in P \cap \partial \Omega_{1}$, and $\|A x\| \leq\|x\|$, for all $x \in P \cap \partial \Omega_{2}$;

(b) $\|A x\| \leq\|x\|$, for all $x \in P \cap \partial \Omega_{1}$, and $\|A x\| \geq\|x\|$, for all $x \in P \cap \partial \Omega_{2}$.

Then, $A$ has at least one fixed point in $P \cap\left(\Omega_{2} \backslash \bar{\Omega}_{1}\right)$.

\section{Preliminaries}

In order to define the solution of problem (1.6), we shall consider the following space.

Let $J^{\prime}=J \backslash\left\{t_{1}, t_{2}, \ldots, t_{n}\right\}$, and

$$
\mathrm{PC}^{1}[0,1]=\left\{x \in C[0,1]:\left.x^{\prime}\right|_{\left(t_{k}, t_{k+1}\right)} \in C\left(t_{k}, t_{k+1}\right), x^{\prime}\left(t_{k}^{-}\right)=x^{\prime}\left(t_{k}\right), \exists x^{\prime}\left(t_{k}^{+}\right), k=1,2, \ldots, m\right\} .
$$


Then $\mathrm{PC}^{1}[0,1]$ is a real Banach space with norm

$$
\|x\|_{\mathrm{PC}^{1}}=\max \left\{\|x\|_{\infty},\left\|x^{\prime}\right\|_{\infty}\right\},
$$

where $\|x\|_{\infty}=\sup _{t \in J}|x(t)|,\left\|x^{\prime}\right\|_{\infty}=\sup _{t \in J}\left|x^{\prime}(t)\right|$.

A function $x \in \mathrm{PC}^{1}[0,1] \cap C^{4}\left(J^{\prime}\right)$ is called a solution of problem (1.6) if it satisfies (1.6).

To establish the existence of multiple positive solutions in $\operatorname{PC}^{1}[0,1] \cap C^{4}\left(J^{\prime}\right)$ of problem (1.6), let us list the following assumptions:

$\left(H_{1}\right) f \in C\left(J \times R^{+}, R^{+}\right), I_{k} \in C\left(R^{+}, R^{+}\right), k=1,2, \ldots, m ;$

$\left(H_{2}\right) g, h \in L^{1}[0,1]$ with

$$
\begin{aligned}
& 0 \leq g(t)<(\tau+1) t^{\tau}, \quad \tau=0,1,2, \ldots, n, \\
& 0 \leq h(t)<t^{\tau}+\frac{\tau}{\tau+1}, \quad \tau=0,1,2, \ldots, n
\end{aligned}
$$

Write

$$
\mu=\int_{0}^{1} g(s) d s, \quad v=\int_{0}^{1} h(s) d s .
$$

From $\left(H_{2}\right)$, it is clear that $\mu \in[0,1), v \in[0,1)$.

We shall reduce problem (1.6) to an integral equation. To this goal, firstly by means of the transformation

$$
\phi_{p}\left(y^{\prime \prime}(t)\right)=-x(t)
$$

we convert problem (1.6) into

$$
\begin{gathered}
x^{\prime \prime}(t)+f(t, y(t))=0, \quad t \in J, \\
x(0)=x(1)=\int_{0}^{1} h(t) x(t) d t, \\
y^{\prime \prime}(t)=-\phi_{q}(x(t)), \quad t \in J, t \neq t_{k}, \\
\left.\Delta y^{\prime}\right|_{t=t_{k}}=-I_{k}\left(y\left(t_{k}\right)\right), \quad k=1,2, \ldots, m, \\
y(0)=y(1)=\int_{0}^{1} g(t) y(t) d t .
\end{gathered}
$$

Lemma 2.1. Assume that $\left(H_{1}\right)$ and $\left(H_{2}\right)$ hold. Then problem (2.6) has a unique solution $x$ given by

$$
x(t)=\int_{0}^{1} H(t, s) f(s, y(s)) d s,
$$


where

$$
\begin{gathered}
H(t, s)=G(t, s)+\frac{1}{1-v} \int_{0}^{1} G(s, \tau) h(\tau) d \tau, \\
G(t, s)= \begin{cases}t(1-s), & 0 \leq t \leq s \leq 1, \\
s(1-t), & 0 \leq s \leq t \leq 1 .\end{cases}
\end{gathered}
$$

Proof. The proof follows by routine calculations.

Write $e(t)=t(1-t)$. Then from (2.9) and (2.10), we can prove that $H(t, s)$ and $G(t, s)$ have the following properties.

Proposition 2.2. If $\left(\mathrm{H}_{2}\right)$ holds, then we have

$$
\begin{gathered}
H(t, s)>0, \quad G(t, s)>0, \quad \text { for } t, s \in(0,1), \\
H(t, s) \geq 0, \quad G(t, s) \geq 0, \quad \text { for } t, s \in J .
\end{gathered}
$$

Proposition 2.3. For $t, s \in[0,1]$, we have

$$
e(t) e(s) \leq G(t, s) \leq G(t, t)=t(1-t)=e(t) \leq \bar{e}=\max _{t \in[0,1]} e(t)=\frac{1}{4}
$$

Proposition 2.4. If $\left(\mathrm{H}_{2}\right)$ holds, then for $t, s \in[0,1]$, we have

$$
\rho e(s) \leq H(t, s) \leq \gamma s(1-s)=\gamma e(s) \leq \frac{1}{4} \gamma,
$$

where

$$
\gamma=\frac{1}{1-v^{\prime}}, \quad \rho=\frac{\int_{0}^{1} e(\tau) h(\tau) d \tau}{1-v}
$$

Proof. By (2.6) and (2.12), we have

$$
\begin{aligned}
H(t, s) & =G(t, s)+\frac{1}{1-v} \int_{0}^{1} G(s, \tau) h(\tau) d \tau \\
& \geq \frac{1}{1-v} \int_{0}^{1} G(s, \tau) h(\tau) d \tau \geq \frac{\int_{0}^{1} e(\tau) h(\tau) d \tau}{1-v} s(1-s) \\
& =\rho e(s), \quad t \in[0,1] .
\end{aligned}
$$


On the other hand, noticing $G(t, s) \leq s(1-s)$, we obtain

$$
\begin{aligned}
H(t, s) & =G(t, s)+\frac{1}{1-v} \int_{0}^{1} G(s, \tau) h(\tau) d \tau \\
& \leq s(1-s)+\frac{1}{1-v} \int_{0}^{1} s(1-s) h(\tau) d \tau \\
& \leq s(1-s)\left[1+\frac{1}{1-v} \int_{0}^{1} h(\tau) d \tau\right] \leq s(1-s) \frac{1}{1-v} \\
& =r e(s), \quad t \in[0,1] .
\end{aligned}
$$

The proof of Proposition 2.4 is complete.

Remark 2.5. From (2.9) and (2.13), we can obtain that

$$
\rho e(s) \leq H(s, s) \leq \gamma s(1-s)=\gamma e(s) \leq \frac{1}{4} \gamma, \quad s \in J .
$$

Lemma 2.6. If $\left(H_{1}\right)$ and $\left(H_{2}\right)$ hold, then problem (2.7) has a unique solution $y$ and $y$ can be expressed in the following form:

$$
y(t)=\int_{0}^{1} H_{1}(t, s) \phi_{q}(x(s)) d s+\sum_{k=1}^{m} H_{1}\left(t, t_{k}\right) I_{k}\left(y\left(t_{k}\right)\right)
$$

where

$$
H_{1}(t, s)=G(t, s)+\frac{1}{1-\mu} \int_{0}^{1} G(s, \tau) g(\tau) d \tau
$$

and $G(t, s)$ is defined in (2.10).

Proof. First suppose that $y \in \mathrm{PC}^{1}[0,1] \cap C^{2}\left(J^{\prime}\right)$ is a solution of problem (2.7).

If $t \in\left[0, t_{1}\right)$, it is easy to see by integration of problem (2.7) that

$$
y^{\prime}(t)=y^{\prime}(0)-\int_{0}^{t} \phi_{q}(x(s)) d s
$$


If $t \in\left[t_{1}, t_{2}\right)$, then integrate from $t_{1}$ to $t$,

$$
\begin{aligned}
y^{\prime}(t) & =y^{\prime}\left(t_{1}^{+}\right)-\int_{t_{1}}^{t} \phi_{q}(x(s)) d s=y^{\prime}\left(t_{1}^{-}\right)+\Delta y^{\prime}\left(t_{1}\right)-\int_{t_{1}}^{t} \phi_{q}(x(s)) d s \\
& =y^{\prime}(0)-\int_{0}^{t_{1}} \phi_{q}(x(s)) d s+\Delta y^{\prime}\left(t_{1}\right)-\int_{t_{1}}^{t} \phi_{q}(x(s)) d s \\
& =y^{\prime}(0)-\int_{0}^{t} \phi_{q}(x(s)) d s-I_{1}\left(y\left(t_{1}\right)\right) .
\end{aligned}
$$

Similarly, if $t \in\left[t_{k}, t_{k+1}\right)$, we have

$$
y^{\prime}(t)=y^{\prime}(0)-\int_{0}^{t} \phi_{q}(x(s)) d s-\sum_{t_{k}<t} I_{k}\left(y\left(t_{k}\right)\right)
$$

Integrating again, we can get

$$
y(t)=y(0)+y^{\prime}(0) t-\int_{0}^{t}(t-s) \phi_{q}(x(s)) d s-\sum_{t_{k}<t} I_{k}\left(y\left(t_{k}\right)\right)\left(t-t_{k}\right) .
$$

Letting $t=1$ in (2.23), we find

$$
y^{\prime}(0)=\int_{0}^{1}(1-s) \phi_{q}(x(s)) d s+\sum_{t_{k}<1} I_{k}\left(y\left(t_{k}\right)\right)\left(1-t_{k}\right) .
$$

Substituting $y(0)=\int_{0}^{1} g(t) y(t) d t$ and (2.24) into (2.23), we obtain

$$
\begin{aligned}
y(t)= & y(0)+\int_{0}^{1} t(1-s) \phi_{q}(x(s)) d s+t \sum_{t_{k}<1} I_{k}\left(y\left(t_{k}\right)\right)\left(1-t_{k}\right) \\
& -\int_{0}^{t}(t-s) \phi_{q}(x(s)) d s-\sum_{t_{k}<t} I_{k}\left(y\left(t_{k}\right)\right)\left(t-t_{k}\right) \\
= & \int_{0}^{1} G(t, s) \phi_{q}(x(s)) d s+\int_{0}^{1} g(t) y(t) d t+\sum_{k=1}^{m} G\left(t, t_{k}\right) I_{k}\left(y\left(t_{k}\right)\right),
\end{aligned}
$$


where

$$
\begin{aligned}
\int_{0}^{1} g(t) y(t) d t= & \int_{0}^{1} g(t)\left[\int_{0}^{1} g(t) y(t) d t+\int_{0}^{1} G(t, s) \phi_{q}(x(s)) d s+\sum_{k=1}^{m} G\left(t, t_{k}\right) I_{k}\left(y\left(t_{k}\right)\right)\right] d t \\
= & \int_{0}^{1} g(t) d t \times \int_{0}^{1} g(t) y(t) d t+\iint_{0}^{1} G(t, s) g(t) \phi_{q}(x(s)) d s d t \\
& +\int_{0}^{1} g(t)\left(\sum_{k=1}^{m} G\left(t, t_{k}\right) I_{k}\left(y\left(t_{k}\right)\right)\right) d t
\end{aligned}
$$

Therefore, we have

$$
\begin{aligned}
& \int_{0}^{1} g(s) y(s) d s= \frac{1}{1-\int_{0}^{1} g(s) d s}\left[\int_{0}^{1}\left(\int_{0}^{1} G(s, r) g(r) d r\right) \phi_{q}(x(s)) d s\right. \\
&\left.+\int_{0}^{1} g(s)\left(\sum_{k=1}^{m} G\left(s, t_{k}\right) I_{k}\left(y\left(t_{k}\right)\right)\right) d s\right], \\
& y(t)=\int_{0}^{1} G(t, s) \phi_{q}(x(s)) d s+\sum_{k=1}^{m} G\left(t, t_{k}\right) I_{k}\left(y\left(t_{k}\right)\right) \\
&+\frac{1}{1-\mu}\left[\int_{0}^{1}\left(\int_{0}^{1} G(s, r) g(r) d r\right) \phi_{q}(x(s)) d s\right. \\
&\left.+\int_{0}^{1} g(s)\left(\sum_{k=1}^{m} G\left(s, t_{k}\right) I_{k}\left(y\left(t_{k}\right)\right)\right) d s\right] .
\end{aligned}
$$

Let

$$
H_{1}(t, s)=G(t, s)+\frac{1}{1-\mu} \int_{0}^{1} G(s, r) g(r) d r .
$$

Then,

$$
y(t)=\int_{0}^{1} H(t, s) \phi_{q}(x(s)) d s+\sum_{k=1}^{m} H\left(t, t_{k}\right) I_{k}\left(y\left(t_{k}\right)\right)
$$

and the proof of sufficient is complete.

Conversely, if $y$ is a solution of (2.18).

Direct differentiation of (2.18) implies, for $t \neq t_{k}$,

$$
y^{\prime}(t)=\int_{0}^{1}(1-s) \phi_{q}(x(s)) d s+\sum_{k=1}^{m} I_{k}\left(y\left(t_{k}\right)\right)\left(1-t_{k}\right)-\int_{0}^{t} \phi_{q}(x(s)) d s-\sum_{t_{k}<t} I_{k}\left(y\left(t_{k}\right)\right) .
$$


Evidently,

$$
\begin{gathered}
y^{\prime \prime}(t)=-\phi_{q}(x(t)) \\
\left.\Delta y^{\prime}\right|_{t=t_{k}}=-I_{k}\left(y\left(t_{k}\right)\right), \quad(k=1,2, \ldots, m), y(0)=y(1)=\int_{0}^{1} g(t) y(t) d t .
\end{gathered}
$$

The Lemma is proved.

Remark 2.7. From (2.19), we can prove that the properties of $H_{1}(t, s)$ are similar to that of $H(t, s)$.

Suppose that $y$ is a solution of problem (1.6). Then from Lemmas 2.6 and 2.1, we have

$$
y(t)=\int_{0}^{1} H_{1}(t, s) \phi_{q}\left(\int_{0}^{1} H(s, \tau) f(\tau, y(\tau)) d \tau\right) d s+\sum_{k=1}^{m} H_{1}\left(t, t_{k}\right) I_{k}\left(y\left(t_{k}\right)\right) .
$$

For the sake of applying Lemma 1.1, we construct a cone in $\mathrm{PC}^{1}[0,1]$ via

$$
K=\left\{x \in \mathrm{PC}^{1}[0,1]: x \geq 0, x(t) \geq \frac{\rho_{1} \rho^{q-1}}{\gamma^{q-1} \gamma_{1}} x(s), t, s \in J\right\}
$$

where

$$
\rho_{1}=\frac{\int_{0}^{1} e(\tau) g(\tau) d \tau}{1-\mu}, \quad \gamma_{1}=\frac{1}{1-\mu}
$$

It is easy to see that $K$ is a closed convex cone of $\mathrm{PC}^{1}[0,1]$.

Define an operator $T: K \rightarrow K$ by

$$
(T y)(t)=\int_{0}^{1} H_{1}(t, s) \phi_{q}\left[\int_{0}^{1} H(s, \tau) f(\tau, y(\tau)) d \tau\right] d s+\sum_{k=1}^{m} H_{1}\left(t, t_{k}\right) I_{k}\left(y\left(t_{k}\right)\right) .
$$

From (2.35), we know that $y \in \operatorname{PC}^{1}[0,1]$ is a solution of problem (1.6) if and only if $y$ is a fixed point of operator $T$.

Definition 2.8 (see [1]). The set $S \subset \mathrm{PC}^{1}[0,1]$ is said to be quasi-equicontinuous in $\mathrm{PC}^{1}[0,1]$ if for any $\varepsilon>0$ there exist $\delta>0$ such that if $u \in S, s, t \in J_{k}(k=1,2, \ldots, m),|s-t|<\delta$, then

$$
|u(s)-u(t)|<\varepsilon,\left|u^{\prime}(s)-u^{\prime}(t)\right|<\varepsilon
$$


We present the following result about relatively compact sets in $\operatorname{PC}^{1}(J)$ which is a consequence of the Arzela-Ascoli Theorem. The reader can find its proof partially in [1].

Lemma 2.9. $S \subset P C^{1}[0,1]$ is relatively compact if and only if $S$ is bounded and quasi-equicontinuous on $P C^{1}[0,1]$.

Write

$$
K_{r, R}=\{x \in K: r<\|x\|<R\},
$$

where $0<r<R$.

Lemma 2.10. Suppose that $\left(H_{1}\right)$ and $\left(H_{2}\right)$ hold. Then $T(K) \subset K$ and $T: K_{r, R} \rightarrow K$ is completely continuous.

Proof. For $y \in K$, it is clear that $T y \geq 0, T y \in \mathrm{PC}^{1}[0,1]$, and

$$
(T y)(t) \leq \gamma^{q-1} \gamma_{1} \int_{0}^{1} e(s) \phi_{q}\left[\int_{0}^{1} e(\tau) f(\tau, y(\tau)) d \tau\right] d s+\gamma_{1} \sum_{k=1}^{m} e\left(t_{k}\right) I_{k}\left(y\left(t_{k}\right)\right)
$$

From (2.35) and Remark 2.5, we obtain the following cases.

Case 1. if $\mu, v \in[0,4 / 5]$, noticing $0 \leq \rho \leq 1$, then we have

$$
\begin{aligned}
(T y)(t) & =\int_{0}^{1} H_{1}(t, s) \phi_{q}\left[\int_{0}^{1} H(s, \tau) f(\tau, y(\tau)) d \tau\right] d s+\sum_{k=1}^{m} H_{1}\left(t, t_{k}\right) I_{k}\left(y\left(t_{k}\right)\right) \\
& \geq \rho_{1} \rho^{q-1} \phi_{q} \int_{0}^{1} e(s)\left[\int_{0}^{1} e(\tau) f(\tau, y(\tau)) d \tau\right] d s+\rho_{1} \sum_{k=1}^{m} e\left(t_{k}\right) I_{k}\left(y\left(t_{k}\right)\right) \\
& \geq \rho_{1} \rho^{q-1} \int_{0}^{1} e(s) \phi_{q}\left[\int_{0}^{1} e(\tau) f(\tau, y(\tau)) d \tau\right] d s+\rho_{1} \rho^{q-1} \sum_{k=1}^{m} e\left(t_{k}\right) I_{k}\left(y\left(t_{k}\right)\right) \\
& =\frac{\rho_{1} \rho^{q-1}}{\gamma^{q-1} \gamma_{1}} \gamma^{q-1} \gamma_{1} \int_{0}^{1} e(s) \phi_{q}\left[\int_{0}^{1} e(\tau) f(\tau, y(\tau)) d \tau\right] d s+\frac{\rho_{1} \rho^{q-1}}{\gamma_{1}} \gamma_{1} \sum_{k=1}^{m} e\left(t_{k}\right) I_{k}\left(y\left(t_{k}\right)\right) \\
& \geq \frac{\rho_{1} \rho^{q-1}}{\gamma^{q-1} \gamma_{1}}\left[\gamma^{q-1} \gamma_{1} \int_{0}^{1} e(s) \phi_{q}\left[\int_{0}^{1} e(\tau) f(\tau, y(\tau)) d \tau\right] d s+\gamma_{1} \sum_{k=1}^{m} e\left(t_{k}\right) I_{k}\left(y\left(t_{k}\right)\right)\right] \\
& \geq \frac{\rho_{1} \rho^{q-1}}{\gamma^{q-1} \gamma_{1}}(T y)(s), \quad t, s \in J .
\end{aligned}
$$


Case 2. if $\mu, v \in[4 / 5,1)$, noticing $\rho \geq 1$ and $\gamma^{q-1}>\rho^{q-1}$, then we have

$$
\begin{aligned}
(T y)(t) & =\int_{0}^{1} H_{1}(t, s) \phi_{q}\left[\int_{0}^{1} H(s, \tau) f(\tau, y(\tau)) d \tau\right] d s+\sum_{k=1}^{m} H_{1}\left(t, t_{k}\right) I_{k}\left(y\left(t_{k}\right)\right) \\
& \geq \rho_{1} \rho^{q-1} \phi_{q} \int_{0}^{1} e(s)\left[\int_{0}^{1} e(\tau) f(\tau, y(\tau)) d \tau\right] d s+\rho_{1} \sum_{k=1}^{m} e\left(t_{k}\right) I_{k}\left(y\left(t_{k}\right)\right) \\
& =\rho_{1} \rho^{q-1} \int_{0}^{1} e(s) \phi_{q}\left[\int_{0}^{1} e(\tau) f(\tau, y(\tau)) d \tau\right] d s+\frac{\rho_{1} \rho^{q-1}}{\rho^{q-1}} \sum_{k=1}^{m} e\left(t_{k}\right) I_{k}\left(y\left(t_{k}\right)\right) \\
& =\frac{\rho_{1} \rho^{q-1}}{\gamma^{q-1} \gamma_{1}} \gamma^{q-1} \gamma_{1} \int_{0}^{1} e(s) \phi_{q}\left[\int_{0}^{1} e(\tau) f(\tau, y(\tau)) d \tau\right] d s+\frac{\rho_{1} \rho^{q-1}}{\gamma_{1} \rho^{q-1}} \gamma_{1} \sum_{k=1}^{m} e\left(t_{k}\right) I_{k}\left(y\left(t_{k}\right)\right) \\
& \geq \frac{\rho_{1} \rho^{q-1}}{\gamma^{q-1} \gamma_{1}}\left[\gamma^{q-1} \gamma_{1} \int_{0}^{1} e(s) \phi_{q}\left[\int_{0}^{1} e(\tau) f(\tau, y(\tau)) d \tau\right] d s+\gamma_{1} \sum_{k=1}^{m} e\left(t_{k}\right) I_{k}\left(y\left(t_{k}\right)\right)\right] \\
& \geq \frac{\rho_{1} \rho^{q-1}}{\gamma^{q-1} \gamma_{1}}(T y)(s), \quad t, s \in J .
\end{aligned}
$$

Therefore, $T(y) \in K$, that is, $T(K) \subset K$. Also, we have $T\left(K_{r, R}\right) \subset K$ since $K_{r, R} \subset K$. Hence we have $T: K_{r, R} \rightarrow K$.

Next, we prove that $T: K_{r, R} \rightarrow K$ is completely continuous.

It is obvious that $T: K_{r, R} \rightarrow K$ is continuous. Now we prove $T$ is relatively compact. Let $B_{r}=\left\{x \in \mathrm{PC}^{1}[0,1] \mid\|x\|_{\mathrm{PC}^{1}} \leq r\right\}$ be a bounded set. Then, for all $x \in B_{r}$, we have

$$
\begin{gathered}
\|T x\|_{\infty} \leq \frac{1}{4} \gamma_{1} \phi_{q}\left(\frac{1}{4} \gamma M\right)+\frac{1}{4} \gamma_{1} m A=r_{0}, \\
\left\|T x^{\prime}\right\|_{\infty} \leq \phi_{q}\left(\frac{1}{4} \gamma M\right)+m A=r_{1} .
\end{gathered}
$$

Therefore $T\left(B_{r}\right)$ is uniformly bounded.

On the other hand, for all $t, s \in J_{k}(k=0,1, \ldots, m)$ with $t<s$, we have

$$
|(T x)(t)-(T x)(s)|=\left|\int_{s}^{t}(T x)^{\prime}(\tau) d \tau\right| \leq r_{1}|t-s| \longrightarrow 0, \quad(s \longrightarrow t)
$$


and by the continuity of $H_{1}^{\prime}(t, s)$, we have

$$
\begin{aligned}
\left|(T x)^{\prime}(t)-(T x)^{\prime}(s)\right|=\mid & \int_{0}^{1}\left[H_{1}^{\prime}(t, r)-H_{1}^{\prime}(s, r)\right] \phi_{q}\left[\int_{0}^{1} H(r, \tau) f(\tau, y(\tau)) d \tau\right] d r \\
& +\sum_{k=1}^{m}\left[H_{1}^{\prime}\left(t, t_{k}\right)-H_{1}^{\prime}\left(s, t_{k}\right)\right] I_{k}\left(y\left(t_{k}\right)\right) \mid \longrightarrow 0, \quad(s \longrightarrow t),
\end{aligned}
$$

and then $T\left(B_{r}\right)$ is quasi-equicontinuous. It follows that $T\left(B_{r}\right)$ is relatively compact on $\mathrm{PC}^{1}[0,1]$ by Lemma 2.9 . So $T$ is completely continuous.

\section{Main Results}

In this section, we apply Lemma 1.1 to establish the existence of positive solutions of problem (1.6). We begin by introducing the following conditions on $f(t, y)$ and $I_{k}(y)$.

$\left(H_{3}\right)$ There exist numbers $0<r<R<+\infty$ such that

$$
\begin{gathered}
f(t, y) \leq \phi_{p}\left(\frac{1}{2 \gamma^{q-1}}(1-\mu) r\right), \quad t \in J, 0 \leq y \leq r, \\
I_{k}(y) \leq \frac{7}{8 m}(1-\mu) r, \quad 0 \leq y \leq r, \\
f(t, y) \geq \phi_{p}\left(\frac{\gamma_{1} \gamma^{q-1}}{\rho_{1}^{2} \rho^{2(q-1)} t_{1}^{q}\left(1-t_{m}\right)^{q}\left(t_{m}-t_{1}\right)^{q}} R\right), \quad t \in J, R \leq y<\infty,
\end{gathered}
$$

where $\mu$ is defined in (2.4), $\rho, \gamma$ are defined in (2.14), respectively, and write

$$
\begin{gathered}
f^{\beta}=\limsup _{y \rightarrow \beta} \max _{t \in J} \frac{f(t, y)}{\phi_{p}(y)}, \quad f_{\beta}=\liminf _{y \rightarrow \beta} \min _{t \in J} \frac{f(t, y)}{\phi_{p}(y)}, \\
I^{\beta}(k)=\limsup _{y \rightarrow \beta} \frac{I_{k}(y)}{y},
\end{gathered}
$$

where $\beta$ denotes $0^{+}$or $+\infty$.

Theorem 3.1. Assume that $\left(H_{1}\right)-\left(H_{3}\right)$ hold. Then problem (1.6) has at least one positive solution $y(t), t \in J$ with

$$
\frac{\rho^{q-1} \rho_{1}}{\gamma_{1} \gamma^{q-1}} r \leq y(t) \leq \frac{\gamma_{1} \gamma^{q-1}}{\rho^{q-1} \rho_{1}} R, \quad t \in J .
$$


Proof. Let $T$ be the cone preserving, completely continuous operator that was defined by (2.35). For $y \in K$ with $\|y\|_{\mathrm{PC}^{1}}=r$, (2.13), (2.19), and (3.1) imply

$$
\begin{aligned}
(T y)(t) & \leq \gamma_{1} \gamma^{q-1} \int_{0}^{1} e(s) \phi_{q}\left(\int_{0}^{1} e(\tau) f(\tau, y(\tau)) d \tau\right) d s+\gamma_{1} \sum_{k=1}^{m} e\left(t_{k}\right) I_{k}\left(y\left(t_{k}\right)\right) \\
& \leq \gamma_{1} \gamma^{q-1} \frac{1}{4}\left(\frac{1}{4}\right)^{q-1} \phi_{q}\left(\int_{0}^{1} f(\tau, y(\tau)) d \tau\right) d s+\frac{1}{4} \gamma_{1} \sum_{k=1}^{m} I_{k}\left(y\left(t_{k}\right)\right) \\
& \leq \gamma_{1} \gamma^{q-1} \frac{1}{4}\left(\frac{1}{4}\right)^{q-1} \phi_{q}\left(\int_{0}^{1} \phi_{p}\left(\frac{1}{2 \gamma^{q-1}}(1-\mu) r\right) d \tau\right) d s+\frac{1}{4} \gamma_{1} m \times \frac{7}{8 m}(1-\mu) r \\
& \leq\left(\frac{1}{4}\right)^{q} \frac{1}{2} r+\frac{7}{32} r \leq \frac{1}{8} r+\frac{7}{32} r<r=\|y\|_{\mathrm{PC}^{1}}, \\
\left|(T y)^{\prime}(t)\right| & \leq \int_{0}^{1}\left|H_{t}^{\prime}(t, s)\right| \phi_{q}\left(\int_{0}^{1} H(s, \tau) f(\tau, y(\tau)) d \tau\right) d s+\sum_{k=1}^{m}\left|H_{t}^{\prime}\left(t, t_{k}\right)\right| I_{k}\left(y\left(t_{k}\right)\right) \\
& \leq \gamma^{q-1}\left(\frac{1}{4}\right)^{q-1} \phi_{q}\left(\int_{0}^{1} f(\tau, y(\tau)) d \tau\right)+\sum_{k=1}^{m} I_{k}\left(y\left(t_{k}\right)\right) \\
& \leq \gamma^{q-1}\left(\frac{1}{4}\right)^{q-1} \phi_{q}\left(\int_{0}^{1}\left(\frac{1}{2 \gamma^{q-1}}(1-\mu) r\right) d \tau\right)+m \times \frac{7}{8 m}(1-\mu) r \\
& \leq \frac{1}{8}(1-\mu) r+\frac{7}{8} r \leq r=\|y\|_{\mathrm{PC}}{ }^{1},
\end{aligned}
$$

where

$$
\begin{gathered}
H_{t}^{\prime}(t, s)=G_{t}^{\prime}(t, s)= \begin{cases}-s & \text { if } 0 \leq s \leq t \leq 1, \\
1-s & \text { if } 0 \leq t \leq s \leq 1,\end{cases} \\
\max _{t, s \in J, t \neq s}\left|H_{t}^{\prime}(t, s)\right|=\max _{t, s \in J, t \neq s}\left|G_{t}^{\prime}(t, s)\right|=1 .
\end{gathered}
$$

Now if we let $\Omega_{1}=\left\{y \in K:\|y\|_{\mathrm{PC}^{1}}<r\right\}$, then (3.5) shows that

$$
\|T y\|_{\mathrm{PC}^{1}} \leq\|y\|_{\mathrm{PC}^{1}}, \quad y \in \partial \Omega_{1} .
$$

Further, let

$$
\begin{gathered}
R_{1}=\frac{\gamma_{1} \gamma^{q-1}}{\rho^{q-1} \rho_{1}} R, \\
\Omega_{2}=\left\{y \in K:\|y\|_{\mathrm{PC}^{1}}<R_{1}\right\} .
\end{gathered}
$$


Then, $y \in K$ and $\|y\|_{\mathrm{PC}^{1}}=R_{1}$ implies

$$
y(t) \geq \frac{\rho_{1} \rho^{q-1}}{\gamma^{q-1} \gamma_{1}} y(s), \quad t, s \in J,
$$

that is,

$$
y(t) \geq \frac{\rho_{1} \rho^{q-1}}{\gamma^{q-1} \gamma_{1}} R_{1}=R, \quad t \in J
$$

Hence, $y(t) \geq R$ for all $t \in J$. Therefore, for all $t \in J$, (3.2) implies

$$
\begin{aligned}
(T y)(t) & \geq \int_{0}^{1} H_{1}(t, s) \phi_{q}\left[\int_{0}^{1} H(s, \tau) f(\tau, y(\tau)) d \tau\right] d s \\
& \geq \rho_{1} \rho^{q-1} \int_{t_{1}}^{t_{m}} e(s) \phi_{q}\left[\int_{t_{1}}^{t_{m}} e(\tau) \phi_{p}\left(\frac{\gamma_{1} \gamma^{q-1}}{\rho_{1}^{2} \rho^{2(q-1)} t_{1}^{q}\left(1-t_{m}\right)^{q}\left(t_{m}-t_{1}\right)^{q}} R\right) d \tau\right] d s \\
& \geq \rho_{1} \rho^{q-1} t_{1}\left(1-t_{m}\right) t_{1}^{q-1}\left(1-t_{m}\right)^{q-1}\left(t_{m}-t_{1}\right)^{q} \times \frac{\gamma_{1} \gamma^{q-1}}{\rho_{1}^{2} \rho^{2(q-1)} t_{1}^{q}\left(1-t_{m}\right)^{q}\left(t_{m}-t_{1}\right)^{q}} R \\
& =\frac{\gamma_{1} \gamma^{q-1}}{\rho_{1} \rho^{q-1}} R=R_{1}=\|y\|_{\mathrm{PC}^{1}{ }^{\prime}}
\end{aligned}
$$

that is, $y \in \partial \Omega_{2}$ implies

$$
\|T y\|_{\mathrm{PC}^{1}} \geq\|y\|_{\mathrm{PC}^{1}}
$$

Applying (b) of Lemma 1.1 to (3.7) and (3.12) yields that $T$ has a fixed point $y \in \bar{\Omega}_{2} \backslash \Omega_{1}$ with $r \leq\|y\|_{\mathrm{PC}^{1}} \leq R_{1}$. Hence, since for $y \in K$ we have $y(t) \geq\left(\rho_{1} \rho^{q-1} / \gamma_{1} \gamma^{q-1}\right) y(s), t, s \in J$, it follows that (3.4) holds. This and Lemma 2.9 complete the proof.

As a special case of Theorem 3.1, we can prove the following results.

Corollary 3.2. Assume that $\left(H_{1}\right)$ and $\left(H_{2}\right)$ hold. If $f^{0}=0, I^{0}(k)=0$, and $f_{\infty}=\infty$, then, for $r>0$ being sufficiently small and $R>0$ being sufficiently large, BVP (1.6) has at least one positive solution $y(t), t \in J$ with property (3.4).

Proof. The proof is similar to that of Theorem 3.1 of [6]. 
In Theorem 3.3, we assume the following condition on $f(t, y)$ and $I_{k}(y)$.

$\left(H_{4}\right)$ There exist numbers $0<r<R<+\infty$ such that

$$
\begin{gathered}
f(t, y) \geq \phi_{p}\left(\frac{\gamma_{1} \gamma^{q-1}}{\rho^{2} \rho_{1}^{2(q-1)} t_{1}^{q}\left(1-t_{m}\right)^{q}\left(t_{m}-t_{1}\right)^{q}} y\right), \quad t \in J, 0 \leq y \leq r, \\
f(t, y) \leq \phi_{p}\left(\frac{1-\mu}{4} y\right), \quad t \in J, \quad R \leq y<\infty, \\
I_{k}(y) \leq \frac{1-\mu}{4 m} y, \quad R \leq y<\infty,
\end{gathered}
$$

where $\rho$ and $\gamma$ are defined in (2.14), and write

$$
\begin{gathered}
M=\max _{t \in J, y \in K,\|y\|_{\mathrm{PC}^{1}}=R} f(t, y), \quad N=\max _{y \in K,\|y\|_{\mathrm{PC}^{1}}=R} I_{k}(y), \\
\eta=\max \left\{M, \phi_{p}\left(\frac{1-\mu}{4}\|y\|_{\mathrm{PC}^{1}}\right)\right\} .
\end{gathered}
$$

Theorem 3.3. Assume that $\left(H_{1}\right),\left(H_{2}\right)$, and $\left(H_{4}\right)$ hold. Then problem (1.6) has at least one positive solution $y(t), t \in J$ with

$$
\frac{\rho^{q-1} \rho_{1}}{\gamma_{1} \gamma^{q-1}} r \leq y(t) \leq \max \left\{2 R, \frac{4}{3(1-\mu)}\left(\gamma^{q-1}\left(\frac{1}{4}\right)^{q-1}(2 \eta)^{q-1}+m N\right)\right\}, \quad \forall t \in J
$$

Proof. For $y \in K$ with $\|y\|_{\mathrm{PC}^{1}}=r$, (3.13) implies

$$
\begin{aligned}
(T y)(t) \geq & \int_{0}^{1} H_{1}(t, s) \phi_{q}\left[\int_{0}^{1} H(s, \tau) f(\tau, y(\tau)) d \tau\right] d s \\
\geq & \rho_{1} \rho^{q-1} \int_{t_{1}}^{t_{m}} e(s) \phi_{q}\left[\int_{t_{1}}^{t_{m}} e(\tau) \phi_{p}\left(\frac{\gamma_{1} \gamma^{q-1}}{\rho_{1}^{2} \rho^{2(q-1)} t_{1}^{q}\left(1-t_{m}\right)^{q}\left(t_{m}-t_{1}\right)^{q}} y(\tau)\right) d \tau\right] d s \\
\geq & \rho_{1} \rho^{q-1} t_{1}\left(1-t_{m}\right) t_{1}^{q-1}\left(1-t_{m}\right)^{q-1}\left(t_{m}-t_{1}\right)^{q} \\
& \times \frac{\gamma_{1} \gamma^{q-1}}{\rho_{1}^{2} \rho^{2(q-1)} t_{1}^{q}\left(1-t_{m}\right)^{q}\left(t_{m}-t_{1}\right)^{q}} \frac{\rho_{1} \rho^{q-1}}{\gamma_{1} \gamma^{q-1}}\|y\|_{\mathrm{PC}^{1}}=\|y\|_{\mathrm{PC}^{1},}
\end{aligned}
$$


that is, $y \in \partial \Omega_{1}$ implies

$$
\|T y\|_{\mathrm{PC}^{1}} \geq\|y\|_{\mathrm{PC}^{1}}
$$

Next, we turn to (3.14) and (3.15). From (3.14), (3.15), and (3.16), we have

$$
f(t, y) \leq M+\phi_{p}\left(\frac{1-\mu}{4} y\right), \quad I_{k}(y) \leq \frac{1-\mu}{4 m} y+N .
$$

Let

$$
\begin{gathered}
R_{2}=\max \left\{2 R, \frac{4}{3(1-\mu)}\left(r^{q-1}\left(\frac{1}{4}\right)^{q-1}(2 \eta)^{q-1}+m N\right)\right\}, \\
\Omega_{3}=\left\{y \in K:\|y\|_{\mathrm{PC}^{1}}<R_{2}\right\} .
\end{gathered}
$$

Thus, for $y \in \partial \Omega_{3}$, we have

$$
\begin{aligned}
(T y)(t) \leq & \gamma_{1} \gamma^{q-1} \int_{0}^{1} e(s) \phi_{q}\left(\int_{0}^{1} e(\tau) f(\tau, y(\tau)) d \tau\right) d s+\gamma_{1} \sum_{k=1}^{m} e\left(t_{k}\right) I_{k}\left(y\left(t_{k}\right)\right) \\
\leq & \gamma_{1} \gamma^{q-1} \frac{1}{4}\left(\frac{1}{4}\right)^{q-1} \phi_{q}\left(\int_{0}^{1} f(\tau, y(\tau)) d \tau\right)+\frac{1}{4} \gamma_{1} \sum_{k=1}^{m} I_{k}\left(y\left(t_{k}\right)\right) \\
\leq & \gamma_{1} \gamma^{q-1} \frac{1}{4}\left(\frac{1}{4}\right)^{q-1} \phi_{q}\left(\int_{0}^{1}\left(M+\phi_{p}\left(\frac{1}{4}(1-\mu) y(\tau)\right)\right) d \tau\right) \\
& +\frac{1}{4} \gamma_{1} m \times\left(N+\frac{1}{4 m}(1-\mu) r\right) \\
\leq & \gamma_{1} \gamma^{q-1} \frac{1}{4}\left(\frac{1}{4}\right)^{q-1} \phi_{q}\left(\int_{0}^{1}(2 \eta) d \tau\right)+\frac{1}{4} \gamma_{1} m \times\left(N+\frac{1}{4 m}(1-\mu)\|y\|_{\mathrm{PC}^{1}}\right) \\
\leq & \gamma_{1} \gamma^{q-1}\left(\frac{1}{4}\right)^{q}(2 \eta)^{q-1}+\frac{1}{16}\|y\|_{\mathrm{PC}^{1}}+\frac{1}{4} \gamma_{1} m \times N \\
\leq & \frac{3}{4}\|y\|_{\mathrm{PC}^{1}}+\frac{7}{32}\|y\|_{\mathrm{PC}^{1}}<\|y\|_{\mathrm{PC}^{1}},
\end{aligned}
$$




$$
\begin{aligned}
\left|(T y)^{\prime}(t)\right| \leq & \int_{0}^{1}\left|H_{t}^{\prime}(t, s)\right| \phi_{q}\left(\int_{0}^{1} H(s, \tau) f(\tau, y(\tau)) d \tau\right) d s+\sum_{k=1}^{m}\left|H_{t}^{\prime}\left(t, t_{k}\right)\right| I_{k}\left(y\left(t_{k}\right)\right) \\
\leq & \gamma^{q-1}\left(\frac{1}{4}\right)^{q-1} \phi_{q}\left(\int_{0}^{1} f(\tau, y(\tau)) d \tau\right)+\sum_{k=1}^{m} I_{k}\left(y\left(t_{k}\right)\right) \\
\leq & \gamma^{q-1}\left(\frac{1}{4}\right)^{q-1} \phi_{q}\left(\int_{0}^{1}\left(M+\phi_{p}\left(\frac{1}{4}(1-\mu) y(\tau)\right)\right) d \tau\right) \\
& +m \times\left(N+\frac{1}{4 m}(1-\mu)\|y\|_{\mathrm{PC}^{1}}\right) \\
\leq & \gamma^{q-1}\left(\frac{1}{4}\right)^{q-1} \phi_{q}\left(\int_{0}^{1} 2 \eta d \tau\right)+m \times\left(N+\frac{1}{4 m}(1-\mu)\|y\|_{\mathrm{PC}^{1}}\right) \\
\leq & \gamma^{q-1}\left(\frac{1}{4}\right)^{q-1} \phi_{q}\left(\int_{0}^{1} 2 \eta d \tau\right)+m \times \frac{1}{4 m}(1-\mu)\|y\|_{\mathrm{PC}^{1}}+m N \\
\leq & \gamma^{q-1}\left(\frac{1}{4}\right)^{q-1}(2 \eta)^{q-1}+m \times \frac{1}{4 m}(1-\mu)\|y\|_{\mathrm{PC}^{1}}+m N \\
\leq & \frac{3}{4}(1-\mu)\|y\|_{\mathrm{PC}^{1}}+\frac{4}{4}\|y\|_{\mathrm{PC}^{1}} \leq(1-\mu)\|y\|_{\mathrm{PC}^{1}} \leq\|y\|_{\mathrm{PC}^{1}} .
\end{aligned}
$$

Then, (3.22) and (3.23) imply

$$
\|T y\|_{\mathrm{PC}^{1}} \leq\|y\|_{\mathrm{PC}^{1}}, \quad y \in \partial \Omega_{3} .
$$

Applying (a) of Lemma 1.1 to (3.19) and (3.24) yields that $T$ has a fixed point $y \in \bar{\Omega}_{3} \backslash$ $\Omega_{1}$ with $r \leq\|y\|_{\mathrm{PC}^{1}} \leq R_{2}$. Hence, since for $y \in K$ we have $y(t) \geq\left(\rho \rho^{q-1} / \gamma_{1} \gamma^{q-1}\right) y(s), t, s \in J$, it follows that (3.17) holds. This and Lemma 2.9 complete the proof.

As a special case of Theorem 3.3, we can prove the following results.

Corollary 3.4. Assume that $\left(H_{1}\right)$ and $\left(H_{2}\right)$ hold. If $f_{0}=\infty$ and $f^{\infty}=0, I^{\infty}(k)=0$; then, for $r>0$ being sufficiently small and $R>0$ being sufficiently large, $B V P(1.6)$ has at least one positive solution $y(t), t \in J$ with property (3.17).

Proof. The proof is similar to that of Theorem 3.2 of [6].

Theorem 3.5. Assume that $\left(H_{1}\right),\left(H_{2}\right),(3.1)$ of $\left(H_{3}\right)$ and (3.14) and (3.15) of $\left(H_{4}\right)$ hold. In addition, letting $f$ and $I_{k}$ satisfy the following condition:

$\left(H_{5}\right)$ There is a $\xi>0$ such that $\left(\rho_{1} \rho^{q-1} / \gamma_{1} \gamma^{q-1}\right) \xi \leq y \leq \xi$ and $t \in J$ implies

$$
f(t, y)>\phi_{p}\left(\frac{1}{\rho_{1} \rho^{q-1} t_{1}^{q}\left(1-t_{m}\right)^{q}\left(t_{m}-t_{1}\right)^{q}} \xi\right),
$$


Then, problem (1.6) has at least two positive solutions $y^{*}(t)$ and $y^{* *}(t)$ with

$$
\frac{\rho}{\gamma} l \leq y^{*}(t)<\xi<y^{* *}(t) \leq L, \quad t \in J,
$$

where $l>0$ and $L>0$ satisfy

$$
0<l<\xi<L .
$$

Proof. If (3.1) of $\left(H_{3}\right)$ holds, similar to the proof of (3.7), we can prove that

$$
\|T y\|_{\mathrm{PC}^{1}} \leq\|y\|_{\mathrm{PC}^{1}}, \quad y \in K,\|y\|_{\mathrm{PC}^{1}}=l
$$

If (3.14) and (3.15) of $\left(H_{4}\right)$ hold, similar to the proof of (3.23), we have

$$
\|T y\|_{\mathrm{PC}^{1}} \leq\|y\|_{\mathrm{PC}^{1}}, \quad y \in K,\|y\|_{\mathrm{PC}^{1}}=L .
$$

Finally, we show that

$$
\|T y\|_{\mathrm{PC}^{1}}>\|y\|_{\mathrm{PC}^{1}}, \quad y \in K,\|y\|_{\mathrm{PC}^{1}}=\xi .
$$

In fact, for $y \in K$ with $\|y\|_{\mathrm{PC}^{1}}=\xi$, then by (2.18), we have

$$
y(t) \geq \frac{\rho_{1} \rho^{q-1}}{\gamma_{1} \gamma^{q-1}}\|y\|_{\mathrm{PC}^{1}}=\frac{\rho_{1} \rho^{q-1}}{\gamma_{1} \gamma^{q-1}} \xi,
$$

and it follows from $\left(H_{5}\right)$ that

$$
\begin{aligned}
(T y)(t) \geq & \int_{0}^{1} H_{1}(t, s) \phi_{q}\left[\int_{0}^{1} H(s, \tau) f(\tau, y(\tau)) d \tau\right] d s \\
> & \rho_{1} \rho^{q-1} \int_{t_{1}}^{t_{m}} e(s) \phi_{q}\left[\int_{t_{1}}^{t_{m}} e(\tau) \phi_{p}\left(\frac{1}{\rho_{1} \rho^{q-1} t_{1}^{q}\left(1-t_{m}\right)^{q}\left(t_{m}-t_{1}\right)^{q}} \xi\right) d \tau\right] d s \\
\geq & \rho_{1} \rho^{q-1} t_{1}\left(1-t_{m}\right) t_{1}^{q-1}\left(1-t_{m}\right)^{q-1}\left(t_{m}-t_{1}\right)^{q} \\
& \times \frac{1}{\rho_{1} \rho^{q-1} t_{1}^{q}\left(1-t_{m}\right)^{q}\left(t_{m}-t_{1}\right)^{q}} \xi \\
= & \xi
\end{aligned}
$$

which implies that (3.30) holds.

Applying Lemma 1.1 to (3.28), (3.29), and (3.30) yields that $T$ has two fixed point $y^{*}$ and $y^{* *}$ with $y^{*} \in K_{\bar{l}, \xi}=\left\{x \in K, l \leq\|x\|_{\mathrm{PC}^{1}}<\xi\right\}$, and $x^{* *} \in K_{\xi, \bar{L}}=\left\{x \in K, \xi<\|x\|_{\mathrm{PC}^{1}} \leq L\right\}$. 
Hence, since for $y^{*} \in K$ we have $y^{*}(t) \geq\left(\rho \rho^{q-1} / \gamma_{1} \gamma^{q-1}\right) y^{*}(s), t, s \in J$, it follows that (3.26) holds. This and Lemma 2.9 complete the proof.

Remark 3.6. Similar to the proof of that of [5], we can prove that problem (1.6) can be generalized to obtain many positive solutions.

\section{Discussion}

In this section, we offer some interesting discussions associated with problem (1.6).

Discussion 1. Generally, it is difficult to obtain the upper and lower bounds of positive solutions for nonlinear higher-order boundary value problems (see, e.g., [33, 34, 39-43, 46, $48,49]$ and their references).

For example, we consider the following problems:

$$
\begin{gathered}
\left(\phi_{p}\left(y^{\prime \prime}(t)\right)\right)^{\prime \prime}=f(t, y(t)), \quad t \in J, t \neq t_{k}, \quad k=1,2, \ldots, m, \\
\left.\Delta y^{\prime}\right|_{t=t_{k}}=-I_{k}\left(y\left(t_{k}\right)\right), \quad k=1,2, \ldots, m, \\
y(0)=0, \quad y(1)=\int_{0}^{1} g(t) y(t) d t, \\
\phi_{p}\left(y^{\prime \prime}(0)\right)=0, \quad \phi_{p}\left(y^{\prime \prime}(1)\right)=\int_{0}^{1} h(t) \phi_{p}\left(y^{\prime \prime}(t)\right) d t .
\end{gathered}
$$

Here $J=[0,1], \phi_{p}(s)$ is $p$-Laplace operator, that is, $\phi_{p}(s)=|s|^{p-2} s, p>1,\left(\phi_{p}\right)^{-1}=\phi_{q}, 1 / p+$ $1 / q=1, f \in C\left(J \times R^{+}, R^{+}\right), I_{k} \in C\left(R^{+}, R^{+}\right), R^{+}=[0,+\infty), t_{k}(k=1,2, \ldots, m)$ (where $m$ is fixed positive integer) are fixed points with $0<t_{1}<t_{2}<\cdots<t_{k}<\cdots<t_{m}<1,\left.\Delta x^{\prime}\right|_{t=t_{k}}=$ $x^{\prime}\left(t_{k}^{+}\right)-x^{\prime}\left(t_{k}^{-}\right)$, where $x^{\prime}\left(t_{k}^{+}\right)$and $x^{\prime}\left(t_{k}^{-}\right)$represent the right-hand limit and left-hand limit of $x^{\prime}(t)$ at $t=t_{k}$, respectively, and $g, h \in L^{1}[0,1]$ is nonnegative.

By means of the transformation (2.5), we can convert problem (4.1) into

$$
\begin{gathered}
x^{\prime \prime}(t)+f(t, y(t))=0, \quad t \in J, \\
x(0)=0, \quad x(1)=\int_{0}^{1} h(t) x(t) d t, \\
y^{\prime \prime}(t)=-\phi_{q}(x(t)), \quad t \in J, \quad t \neq t_{k}, \\
\left.\Delta y^{\prime}\right|_{t=t_{k}}=-I_{k}\left(y\left(t_{k}\right)\right), \quad k=1,2, \ldots, m, \\
y(0)=0, \quad y(1)=\int_{0}^{1} g(t) y(t) d t .
\end{gathered}
$$


Using the similar proof of that of Lemmas 2.1 and 2.6, we can obtain the following results. In addition, if we replace $\mu, v$ by $\mu^{*}, v^{*}$ in $\left(H_{2}\right)$, respectively, then we obtain $\left(H_{2}^{*}\right)$, where

$$
\mu^{*}=\int_{0}^{1} s g(s) d s, \quad v^{*}=\int_{0}^{1} \operatorname{sh}(s) d s
$$

Lemma 4.1. If $\left(H_{1}\right)$ and $\left(H_{2}^{*}\right)$ hold, then BVP (4.2) has a unique solution $x$ and $x$ can be expressed in the form

$$
x(t)=\int_{0}^{1} H^{*}(t, s) f(s, y(s)) d s,
$$

where

$$
H^{*}(t, s)=G(t, s)+\frac{t}{1-\int_{0}^{1} \operatorname{sh}(s) d s} \int_{0}^{1} G((s, \tau) h \tau) d \tau
$$

$G(t, s)$ is defined in (2.10).

Lemma 4.2. If $\left(H_{1}\right)$ and $\left(H_{2}^{*}\right)$ hold, then BVP (4.3) has a unique solution $y$ and $y$ can be expressed in the form

$$
y(t)=\int_{0}^{1} H_{1}^{*}(t, s) \phi_{q}(x(s)) d s+\sum_{k=1}^{m} H_{1}^{*}\left(t, t_{k}\right) I_{k}\left(y\left(t_{k}\right)\right),
$$

where

$$
H_{1}^{*}(t, s)=G(t, s)+\frac{t}{1-\int_{0}^{1} s g(s) d s} \int_{0}^{1} G(s, \tau) g(\tau) d \tau
$$

It is not difficult to prove that $H^{*}(t, s)$ and $H_{1}^{*}(t, s)$ have the similar properties to that of $H(t, s)$ and $H_{1}(t, s)$. But for $t \in J, H^{*}(t, s)$ and $H_{1}^{*}(t, s)$ have no property (2.13). In fact, if $t \in\left[t_{1}, t_{m}\right]$, then we can prove that $H^{*}(t, s)$ and $H_{1}^{*}(t, s)$ have the following properties.

Proposition 4.3. If $\left(H_{2}^{*}\right)$ holds, then for $t \in\left[t_{1}, t_{m}\right], s \in[0,1]$, we have

$$
\begin{aligned}
& \rho^{*} e(s) \leq H^{*}(t, s) \leq \gamma^{*} s(1-s)=\gamma^{*} e(s) \leq \frac{1}{4} \gamma^{*}, \\
& \rho_{1}^{*} e(s) \leq H_{1}^{*}(t, s) \leq \gamma_{1}^{*} s(1-s)=\gamma_{1}^{*} e(s) \leq \frac{1}{4} \gamma_{1}^{*},
\end{aligned}
$$


where

$$
\begin{array}{ll}
r^{*}=\frac{1+\int_{0}^{1}\left(t_{m}-\tau\right) g(\tau) d \tau}{1-\mu^{*}}, & \rho^{*}=\frac{\int_{0}^{1} e(\tau) g(\tau) d \tau}{1-\mu^{*}} t_{1}\left(1-t_{m}\right), \\
r_{1}^{*}=\frac{1+\int_{0}^{1}\left(t_{m}-\tau\right) h(\tau) d \tau}{1-v^{*}}, & \rho_{1}^{*}=\frac{\int_{0}^{1} e(\tau) h(\tau) d \tau}{1-v^{*}} t_{1}\left(1-t_{m}\right) .
\end{array}
$$

Proof. We only consider (4.9). By (4.3), we have

$$
\begin{aligned}
H^{*}(t, s) & =G(t, s)+\frac{t}{1-\mu^{*}} \int_{0}^{1} G(s, \tau) g(\tau) d \tau \\
& \geq \frac{t_{1}}{1-\mu^{*}} \int_{0}^{1} G(s, \tau) g(\tau) d \tau \\
& \geq \frac{\int_{0}^{1} e(\tau) g(\tau) d \tau}{1-\mu^{*}} s(1-s) t_{1}\left(1-t_{m}\right) \\
& =\rho^{*} e(s), \quad t \in\left[t_{1}, t_{m}\right], s \in[0,1] .
\end{aligned}
$$

On the other hand, noticing $G(t, s) \leq s(1-s)$, we obtain

$$
\begin{aligned}
H^{*}(t, s) & =G(t, s)+\frac{t}{1-\mu^{*}} \int_{0}^{1} G(s, \tau) g(\tau) d \tau \\
& \leq s(1-s)+\frac{t_{m}}{1-\mu^{*}} \int_{0}^{1} s(1-s) g(\tau) d \tau \\
& \leq s(1-s)\left[1+\frac{t_{m}}{1-\mu^{*}} \int_{0}^{1} g(\tau) d \tau\right] \\
& \leq s(1-s) \frac{1+\int_{0}^{1}\left(t_{m}-\tau\right) g(\tau) d \tau}{1-\mu^{*}} \\
& =\gamma^{*} e(s), \quad t \in[0,1] .
\end{aligned}
$$

Similarly, we can prove that (4.10) holds, too.

Remark 4.4. Since $\mu^{*} \in[0,1), \gamma^{*}>0$.

From (4.9) and (4.10), we can only define a cone $K^{*}$ by

$$
K^{*}=\left\{y \in \mathrm{PC}^{1}[0,1]: y \geq 0, y(t) \geq \frac{\rho_{1}^{*}\left(\rho^{*}\right)^{q-1}}{\gamma_{1}^{*}\left(\gamma^{*}\right)^{q-1}} y(s), t \in\left[t_{1}, t_{m}\right], s \in J\right\}
$$

which implies that we cannot obtain the lower bounds for the positive solutions of problem (4.1). 


\section{Example}

To illustrate how our main results can be used in practice, we present an example.

Example 5.1. Consider the following boundary value problem:

$$
\begin{gathered}
\left(\phi_{3}\left(y^{\prime \prime}(t)\right)\right)^{\prime \prime}=f(t, y(t)), \quad t \in J, t \neq \frac{1}{3}, t \neq \frac{2}{3}, \\
\left.\Delta y^{\prime}\right|_{t=1 / 3}=-\frac{1}{32} y\left(\frac{1}{3}\right) \\
\left.\Delta y^{\prime}\right|_{t=2 / 3}=-\frac{1}{32} y\left(\frac{2}{3}\right) \\
y(0)=y(1)=\int_{0}^{1} \frac{1}{2} y(t) d t \\
\phi_{3}\left(y^{\prime \prime}(0)\right)=\phi_{3}\left(y^{\prime \prime}(1)\right)=\int_{0}^{1} t \phi_{3}\left(y^{\prime \prime}(t)\right) d t
\end{gathered}
$$

where $t_{1}=1 / 3, t_{2}=2 / 3, m=2, p=3, q=3 / 2, g(t)=1 / 2, h(t)=t$, and

$$
f(t, y)= \begin{cases}\frac{1}{256} y^{2}, & 0 \leq y \leq 1 \\ \left(5668704-\frac{1}{256}\right) y+\left(\frac{2}{256}-5668704\right), & 1 \leq y \leq 2 \\ 5668704 y^{2}, & y \geq 2 .\end{cases}
$$

Conclusion. Equation(5.1) has at least one positive solution $y(t)$ for $t \in J$ with

$$
\frac{1}{12 \sqrt{12}} \leq y(t) \leq 24 \sqrt{12}
$$

Proof. By simple computation, we have $\mu=1 / 2, v=1 / 2, \gamma=2, \rho=1 / 6, \gamma_{1}=2$, and $\rho_{1}=$ $1 / 6$. Select $r=1$, and $R=2$, then for $0<r<R<+\infty$, we have

$$
\begin{gathered}
f(t, y) \leq \frac{1}{256}, \quad \forall 0 \leq y \leq 1, \\
I_{k}(y)=\frac{1}{32} y \leq \frac{7}{32}, \quad \forall 0 \leq y \leq 1, \\
f(t, y) \geq 5668704, \quad \forall y \geq 2 .
\end{gathered}
$$

By Theorem 3.1, (5.1) has a positive solution $y(t)$ with $1 / 12 \sqrt{12} \leq y(t) \leq 24 \sqrt{12}$. 


\section{Acknowledgments}

The authors thank the referee for his/her careful reading of the manuscript and useful suggestions. This work is sponsored by the Funding Project for Academic Human Resources Development in Institutions of Higher Learning under the Jurisdiction of Beijing Municipality (PHR201008430), the Scientific Research Common Program of Beijing Municipal Commission of Education (KM201010772018), and Beijing Municipal Education Commission (71D0911003).

\section{References}

[1] V. Lakshmikantham, D. D. Baĭnov, and P. S. Simeonov, Theory of Impulsive Differential Equations, vol. 6 of Series in Modern Applied Mathematics, World Scientific, Singapore, 1989.

[2] M. Benchohra, J. Henderson, and S. Ntouyas, Impulsive Differential Equations and Inclusions, vol. 2 of Contemporary Mathematics and Its Applications, Hindawi Publishing Corporation, New York, NY, USA, 2006.

[3] B. Ahmad and S. Sivasundaram, "Existence of solutions for impulsive integral boundary value problems of fractional order," Nonlinear Analysis: Hybrid Systems, vol. 4, no. 1, pp. 134-141, 2010.

[4] D. D. Bănov and P. S. Simeonov, Systems with Impulse Effect, Ellis Horwood, Chichester, UK, 1989.

[5] A. M. Samőlenko and N. A. Perestyuk, Impulsive Differential Equations, vol. 14, World Scientific, Singapore, 1995.

[6] J. Yan, "Existence of positive periodic solutions of impulsive functional differential equations with two parameters," Journal of Mathematical Analysis and Applications, vol. 327, no. 2, pp. 854-868, 2007.

[7] M. Feng and D. Xie, "Multiple positive solutions of multi-point boundary value problem for secondorder impulsive differential equations," Journal of Computational and Applied Mathematics, vol. 223, no. 1, pp. 438-448, 2009.

[8] J. J. Nieto, "Basic theory for nonresonance impulsive periodic problems of first order," Journal of Mathematical Analysis and Applications, vol. 205, no. 2, pp. 423-433, 1997.

[9] J. J. Nieto, "Impulsive resonance periodic problems of first order," Applied Mathematics Letters, vol. 15, no. 4, pp. 489-493, 2002.

[10] D. Guo, "Multiple positive solutions for first order nonlinear impulsive integro-differential equations in a Banach space," Applied Mathematics and Computation, vol. 143, no. 2-3, pp. 233-249, 2003.

[11] X. Liu and D. Guo, "Periodic boundary value problems for a class of second-order impulsive integrodifferential equations in Banach spaces," Journal of Mathematical Analysis and Applications, vol. 216, no. 1, pp. 284-302, 1997.

[12] R. P. Agarwal and D. O'Regan, "Multiple nonnegative solutions for second order impulsive differential equations," Applied Mathematics and Computation, vol. 114, no. 1, pp. 51-59, 2000.

[13] B. Liu and J. Yu, "Existence of solution of $m$-point boundary value problems of second-order differential systems with impulses," Applied Mathematics and Computation, vol. 125, no. 2-3, pp. 155$175,2002$.

[14] R. P. Agarwal, D. Franco, and D. O'Regan, "Singular boundary value problems for first and second order impulsive differential equations," Aequationes Mathematicae, vol. 69, no. 1-2, pp. 83-96, 2005.

[15] X. Lin and D. Jiang, "Multiple positive solutions of Dirichlet boundary value problems for second order impulsive differential equations," Journal of Mathematical Analysis and Applications, vol. 321, no. 2, pp. 501-514, 2006.

[16] T. Jankowski, "Positive solutions of three-point boundary value problems for second order impulsive differential equations with advanced arguments," Applied Mathematics and Computation, vol. 197, no. 1, pp. 179-189, 2008.

[17] T. Jankowski, "Positive solutions to second order four-point boundary value problems for impulsive differential equations," Applied Mathematics and Computation, vol. 202, no. 2, pp. 550-561, 2008.

[18] M. Feng, B. Du, and W. Ge, "Impulsive boundary value problems with integral boundary conditions and one-dimensional p-Laplacian," Nonlinear Analysis: Theory, Methods E Applications, vol. 70, no. 9, pp. 3119-3126, 2009.

[19] J. M. Gallardo, "Second-order differential operators with integral boundary conditions and generation of analytic semigroups," Rocky Mountain Journal of Mathematics, vol. 30, no. 4, pp. 1265 1291, 2000. 
[20] G. L. Karakostas and P. Ch. Tsamatos, "Multiple positive solutions of some Fredholm integral equations arisen from nonlocal boundary-value problems," Electronic Journal of Differential Equations, vol. 2002, no. 30, pp. 1-17, 2002.

[21] A. Lomtatidze and L. Malaguti, "On a nonlocal boundary value problem for second order nonlinear singular differential equations," Georgian Mathematical Journal, vol. 7, no. 1, pp. 133-154, 2000.

[22] C. Corduneanu, Integral Equations and Applications, Cambridge University Press, Cambridge, UK, 1991.

[23] R. P. Agarwal and D. O'Regan, Infinite Interval Problems for Differential, Difference and Integral Equations, Kluwer Academic Publishers, Dordrecht, The Netherlands, 2001.

[24] J. R. L. Webb and G. Infante, "Positive solutions of nonlocal boundary value problems involving integral conditions," Nonlinear Differential Equations and Applications, vol. 15, no. 1-2, pp. 45-67, 2008.

[25] J. R. L. Webb and G. Infante, "Non-local boundary value problems of arbitrary order," Journal of the London Mathematical Society, vol. 79, no. 1, pp. 238-258, 2009.

[26] J. R. L. Webb and G. Infante, "Positive solutions of nonlocal boundary value problems: a unified approach," Journal of the London Mathematical Society, vol. 74, no. 3, pp. 673-693, 2006.

[27] B. Ahmad and J. J. Nieto, "The monotone iterative technique for three-point second-order integrodifferential boundary value problems with p-Laplacian," Boundary Value Problems, vol. 2007, Article ID 57481, 9 pages, 2007.

[28] B. Ahmad and J. J. Nieto, "Existence results for nonlinear boundary value problems of fractional integrodifferential equations with integral boundary conditions," Boundary Value Problems, vol. 2009, Article ID 708576, 11 pages, 2009.

[29] B. Ahmad, A. Alsaedi, and B. S. Alghamdi, "Analytic approximation of solutions of the forced Duffing equation with integral boundary conditions," Nonlinear Analysis: Theory, Methods E Applications, vol. 9, no. 4, pp. 1727-1740, 2008.

[30] M. Feng, D. Ji, and W. Ge, "Positive solutions for a class of boundary-value problem with integral boundary conditions in Banach spaces," Journal of Computational and Applied Mathematics, vol. 222, no. 2, pp. 351-363, 2008.

[31] X. Zhang, M. Feng, and W. Ge, "Multiple positive solutions for a class of $m$-point boundary value problems," Applied Mathematics Letters, vol. 22, no. 1, pp. 12-18, 2009.

[32] M. Feng and W. Ge, "Positive solutions for a class of $m$-point singular boundary value problems," Mathematical and Computer Modelling, vol. 46, no. 3-4, pp. 375-383, 2007.

[33] X. Zhang, M. Feng, and W. Ge, "Symmetric positive solutions for $p$-Laplacian fourth-order differential equations with integral boundary conditions," Journal of Computational and Applied Mathematics, vol. 222, no. 2, pp. 561-573, 2008.

[34] X. Zhang, M. Feng, and W. Ge, "Existence results for nonlinear boundary-value problems with integral boundary conditions in Banach spaces," Nonlinear Analysis: Theory, Methods E Applications, vol. 69, no. 10, pp. 3310-3321, 2008.

[35] Z. Yang, "Positive solutions of a second-order integral boundary value problem," Journal of Mathematical Analysis and Applications, vol. 321, no. 2, pp. 751-765, 2006.

[36] R. Ma, "Positive solutions for multipoint boundary value problem with a one-dimensional $p$ Laplacian," Computational E Applied Mathematics, vol. 42, pp. 755-765, 2001.

[37] Z. Bai, B. Huang, and W. Ge, "The iterative solutions for some fourth-order $p$-Laplace equation boundary value problems," Applied Mathematics Letters, vol. 19, no. 1, pp. 8-14, 2006.

[38] B. Liu, L. Liu, and Y. Wu, "Positive solutions for singular second order three-point boundary value problems," Nonlinear Analysis: Theory, Methods \& Applications, vol. 66, no. 12, pp. 2756-2766, 2007.

[39] X. Zhang and L. Liu, "A necessary and sufficient condition for positive solutions for fourth-order multi-point boundary value problems with $p$-Laplacian," Nonlinear Analysis: Theory, Methods $\mathcal{E}$ Applications, vol. 68, no. 10, pp. 3127-3137, 2008.

[40] A. R. Aftabizadeh, "Existence and uniqueness theorems for fourth-order boundary value problems," Journal of Mathematical Analysis and Applications, vol. 116, no. 2, pp. 415-426, 1986.

[41] Z. Bai and H. Wang, "On positive solutions of some nonlinear fourth-order beam equations," Journal of Mathematical Analysis and Applications, vol. 270, no. 2, pp. 357-368, 2002.

[42] R. Ma and H. Wang, "On the existence of positive solutions of fourth-order ordinary differential equations," Applicable Analysis, vol. 59, no. 1-4, pp. 225-231, 1995.

[43] L. Liu, X. Zhang, and Y. Wu, "Positive solutions of fourth order four-point boundary value problems with $p$-Laplacian operator," Journal of Mathematical Analysis and Applications, vol. 326, no. 2, pp. 1212 1224, 2007. 
[44] P. Kang, Z. Wei, and J. Xu, "Positive solutions to fourth-order singular boundary value problems with integral boundary conditions in abstract spaces," Applied Mathematics and Computation, vol. 206, no. 1, pp. 245-256, 2008.

[45] J. R. L. Webb, G. Infante, and D. Franco, "Positive solutions of nonlinear fourth-order boundary-value problems with local and non-local boundary conditions," Proceedings of the Royal Society of Edinburgh, vol. 138, no. 2, pp. 427-446, 2008.

[46] H. Ma, "Symmetric positive solutions for nonlocal boundary value problems of fourth order," Nonlinear Analysis: Theory, Methods E Applications, vol. 68, no. 3, pp. 645-651, 2008.

[47] D. Guo and V. Lakshmikantham, Nonlinear Problems in Abstract Cones, vol. 5, Academic Press, Boston, Mass, USA, 1988.

[48] P. W. Eloe and B. Ahmad, "Positive solutions of a nonlinear $n$th order boundary value problem with nonlocal conditions," Applied Mathematics Letters, vol. 18, no. 5, pp. 521-527, 2005.

[49] X. Hao, L. Liu, and Y. Wu, "Positive solutions for nonlinear $n$ th-order singular nonlocal boundary value problems," Boundary Value Problems, vol. 2007, Article ID 74517, 10 pages, 2007. 